CLINICAL STUDY

\title{
Functional magnetic resource imaging assessment of altered brain function in hypothyroidism during working memory processing
}

Xiao-Song He, Ning Ma, Zhong-Lin Pan $^{1}$, Zhao-Xin Wang ${ }^{2}$, Nan Li, Xiao-Chu Zhang, Jiang-Ning Zhou, De-Fa Zhu ${ }^{3}$ and Da-Ren Zhang

CAS Key Laboratory of Brain Function and Disease and, School of Life Sciences, University of Science and Technology of China, Huangshan Road 443, Hefei 230027, Anhui, China, ${ }^{1}$ Department of Radiology, PLA 105 Hospital, Hefei 230031, Anhui, China, ${ }^{2}$ Key Laboratory of Brain Functional Genomics, Ministry of Education, Institute of Cognitive Neuroscience, East China Normal University, Shanghai 200062, China and ${ }^{3}$ Department of Endocrinology, Anhui Geriatric Institute, The First Affiliated Hospital of Anhui Medical University, Jixi Road 218, Hefei 230022, Anhui, China

(Correspondence should be addressed to DR Zhang; Email: drzhang@ustc.edu.cn; DF Zhu; Email: zdfa0168@sina.com)

\begin{abstract}
Context: Hypothyroidism is related to multiple cognitive deficits including working memory dysfunction, of which the underlying neural correlates were rarely studied. In this study, the impact of hypothyroidism on neural circuits involved in working memory processing was explored by functional magnetic resource imaging (fMRI).

Design: Using fMRI, we conducted a longitudinal study investigating alterations of brain function during a working memory task, the four-digit backward recall (BR) and forward recall (FR), in hypothyroid patients and controls.

Methods: fMRI scan was used in 13 female patients at two time points: before and after having been treated with levothyroxine $\left(\mathrm{L}_{-} \mathrm{T}_{4}\right)$ for $\sim 6$ months, and 12 matched euthyroid controls were also scanned. Wechsler Memory Scale-Chinese Revision was used to assess the memory states of each participant.

Results: The hypothyroid patients showed poorer memory states than that in controls. Furthermore, significant differences of task-induced deactivation (TID, task-dependent decreases in neural activity relative to rest) between patients and controls were found in the bilateral medial prefrontal cortices, posterior cingulate cortices, and left inferior partial lobule $(P<0.05)$. These regions were considered as parts of a task-negative network, namely the default mode network (DMN). Concretely, relative to controls, patients showed diminished TID during BR in contrast to FR. After the ${\mathrm{L}-\mathrm{T}_{4}}_{4}$ treatment, neither the poor memory states nor the alteration of TID was detectable in patients.

Conclusion: Hypothyroidism is related to alterations of TID within DMN regions during working memory processing. These exploratory findings may imply potential neural correlates in hypothyroidism-related cognitive deficits and their recoveries.
\end{abstract}

European Journal of Endocrinology 164 951-959

\section{Introduction}

Thyroid hormone plays a critical role in adult brain, influencing both mood and cognition $(1,2)$. It is well accepted that hypothyroidism is related to pathological alterations of thyroid hormone distributions and functioning in both hippocampus and cerebral cortex $(1,3,4)$. There are various adverse effects of hypothyroidism on cerebral-dependent neurocognitive functions $(1,2,5-7)$ including working memory $(8,9)$, which is the executive and attentional control of short-term memory providing for temporal storage and online manipulation of information (10). Working memory is considered to be the core of many other cognitive functions and vital for general human intelligence $(10,11)$. Furthermore, specific deficit in working memory was found even at the mild stage of the disease (subclinical hypothyroidism) $(9,12)$. Therefore, working memory may be one of the primarily affected cerebral-dependent functions in hypothyroidism. However, the underlying neural substrate of such dysfunction remains to be explored.

Within decades, a new perspective on investigating the disease-brain interaction has been provided through neuroimaging approaches, such as functional magnetic resource imaging (fMRI) (13-15). It is a noninvasive technique that evaluates neural activity by measuring changes in the magnetic field associated with the deoxygenation of hemoglobin, namely the blood oxygen-level dependence (BOLD) signal. fMRI 
scanning during certain conditions, such as performing a cognitive task, is used to reveal regional brain function and its disease-related alteration. Using fMRI, former studies had demonstrated two separate brain networks involved in working memory. Of the two networks, one is the task-positive network, including a series of frontal-partial brain regions showing raised BOLD signal, or the so-called task-induced activation (TIA, task-dependent increases in neural activity) $(16,17)$. The other is the task-negative network, including a series of medial frontal-partial brain regions showing reduced BOLD signal, or the so-called task-induced deactivation (TID, task-dependent decreases in neural activity) (18-20).

Accordingly, in order to investigate the impact of hypothyroidism on neural circuits involved in working memory, we conducted an fMRI study in newly diagnosed hypothyroid adults performing a working memory task, the four-digit backward recall (BR) and forward recall (FR) paradigm. Both TIA and TID during the task were compared between patients and controls to explore the potential hypothyroidism-related alteration in brain function. Post-scan psychometric evaluation using Wechsler Memory Scale-Chinese Revision (WMS-CR) was also employed to assess general memory function, including working memory function $(21,22)$. Moreover, all patients were investigated after having been treated with levothyroxine $\left(\mathrm{L}-\mathrm{T}_{4}\right)$ for $\sim 6$ months, again, to examine the restoration of the altered brain functions.

\section{Patients and methods}

\section{Participants}

A total of 25 right-handed women, 13 patients with adult-onset hypothyroidism due to Hashimoto's thyroiditis and 12 voluntary euthyroid controls (ageand education-matched controls, Table 1), participated in this study. All patients were newly diagnosed with elevated TSH and abnormally low free $\mathrm{T}_{4}\left(\mathrm{fT}_{4}\right)$ and free triiodothyronine $\left(\mathrm{fT}_{3}\right)$ levels, and were recruited from the Division of Endocrinology in The First Affiliated Hospital of Anhui Medical University during 20072009. No participant was post-menopausal. During the entire study, neither of them was under pregnancy nor using oral contraceptives or other medications that were known to affect cognition. No one has reported any previous history of ischemic heart disease, stroke, diabetes, head injury, epilepsy, psychiatric illness, significant visual impairment, or endocrine diseases other than hypothyroidism. This study was approved by the human subjects review committee of The First Affiliated Hospital of Anhui Medical University. Written informed consents were obtained from all participants at recruiting.
Table 1 Comparison of clinical information and psychometric evaluations for hypothyroid and control participants (mean \pm s.D.). Serum hormone levels distributed range: euthyroid controls, TSH $(0.9-3.1 \mathrm{mIU} / \mathrm{l}) ; \mathrm{fT}_{4}(12.6-21.9 \mathrm{pmol} / \mathrm{l}) ; \mathrm{fT}_{3}$ (3.8-5.8 pmol/l). Pre-treatment patients, TSH $(20.7->150 \mathrm{mIU} / \mathrm{l}) ; \mathrm{fT}_{4}$ (3.5-9.7 pmol/l); $\mathrm{fT}_{3}(0.4-3.3 \mathrm{pmol} / \mathrm{l})$. Post-treatment patients, TSH (0.5-4.1 mIU/l); $\mathrm{fT}_{4}(15.6-22.5 \mathrm{pmol} / \mathrm{l}) ; \mathrm{fT}_{3}(3.7-5.8 \mathrm{pmol} / \mathrm{l})$.

\begin{tabular}{|c|c|c|c|}
\hline & \multirow{2}{*}{$\begin{array}{c}\text { Euthyroid } \\
\text { controls } \\
(n=12)\end{array}$} & \multicolumn{2}{|c|}{ Hypothyroid patients $(n=13)$} \\
\hline & & Pre-treatment & Post-treatment \\
\hline Age (years) & $29.3 \pm 6.7$ & $29.4 \pm 6.3$ & \\
\hline Education (years) & $12.8 \pm 3.0$ & $12.0 \pm 3.2$ & \\
\hline TSH (mlU/l) & $1.7 \pm 0.7$ & $102.6 \pm 57.8^{\dagger}$ & $1.8 \pm 1.0$ \\
\hline $\mathrm{fT}_{4}(\mathrm{pmol} / \mathrm{l})$ & $16.9 \pm 2.8$ & $7.2 \pm 1.9^{\dagger}$ & $18.3 \pm 2.5^{\pi}$ \\
\hline $\mathrm{fT}_{3}(\mathrm{pmol} / \mathrm{l})$ & $4.6 \pm 0.7$ & $2.3 \pm 0.9^{\dagger}$ & $4.6 \pm 0.6^{\pi}$ \\
\hline$M Q$ & $124.4 \pm 14.1$ & $100.2 \pm 25.1^{*}$ & $121.0 \pm 13.6^{\prime \prime}$ \\
\hline Numeric span & $14.4 \pm 3.2$ & $12.3 \pm 2.7^{\ddagger}$ & $13.5 \pm 3.0^{\S}$ \\
\hline
\end{tabular}

$\mathrm{fT}_{4}$, free thyroxine; $\mathrm{fT}_{3}$, free triiodothyronine; $\mathrm{MQ}$, memory quotient. Significant differences revealed by independent $t$-test between patients and controls, ${ }^{*} P<0.01,{ }^{\dagger} P<0.001$; ${ }^{\ddagger}$ marginal significant, $P=0.090$. Significant differences revealed by paired $t$-test between pre- and post-treatment patients, ${ }^{\S} P<0.05, " ~ P P<0.01,{ }^{\top} P<0.001$.

\section{Experimental design}

We conducted a longitudinal study that included two identical experimental sessions of fMRI scan and psychometric evaluation for each patient. Of the two sessions, one was performed before treatment. All patients were then treated with $\mathrm{L}_{-} \mathrm{T}_{4}$ immediately, and became euthyroid after 1 month. After that, they were treated continuously to maintain the euthyroid states. The second session was performed after 6 months of the replacement therapy to investigate a stabilized treating effect. Between the two sessions, no treatment other than $\mathrm{L}_{-} \mathrm{T}_{4}$ was employed for any patient. All the controls participated only in one experimental session. Each participant took part in her experimental session during the first 10-15 days after onset of menstrual bleeding.

\section{Hormone level assessment and psychometric evaluation}

Serum hormone levels were measured for each participant 1-day before each session. Serum $\mathrm{fT}_{3}$, $\mathrm{fT}_{4}$, and TSH levels were measured by immunoassay using direct, chemiluminometric technology (ADVIA Ceutaur FT3, FrT4, and TSH-3, respectively, Siemens Healthcare Diagnostics Inc., New York, NY, USA) with a sensitivity of $0.3 \mathrm{pmol} / \mathrm{l}, 1.3 \mathrm{pmol} / \mathrm{l}$ and $0.004 \mathrm{mIU} / \mathrm{l}$ respectively. The normal reference ranges were $3.5-6.5 \mathrm{pmol} / \mathrm{l}$ for $\mathrm{fT}_{3}, 11.5-22.7 \mathrm{pmol} / \mathrm{l}$ for $\mathrm{fT}_{4}$, and $0.35-5.5 \mathrm{mIU} / \mathrm{l}$ for TSH.

General memory states were assessed with the WMS$\mathrm{CR}$, a neurocognitive battery designed for the assessment of memory function by providing an overall memory quotient (MQ) $(21,22)$. A subtest of the 
WMS-CR, the numeric span, which stands for the ageadjusted score of the sum of digital forward and backward span, was particularly evaluated between the groups to assess working memory function (23).

\section{FMRI four-digit BR and FR task}

All participants performed four-digit BR and FR trials (24) during the fMRI scan (Fig. 1). Every trial started with a fixation point displayed for $1 \mathrm{~s}$ in the center of the screen. Subsequently, four digits (selected from 1 to 9 without repetition, $1 \mathrm{~s} /$ digit) were presented sequentially at the location of fixation. After the last digit, a cue was presented to instruct the participants to recall the digits either in a forward or in a backward order by writing them on a sheet of paper. The participants were instructed to keep their heads still and not to watch while writing. An author stood near the scanner and made the recording paper available after each trial. The cue lasted for $1 \mathrm{~s}$. Then, the screen remained blank until the onset of the next trial's fixation point. The total time allocated to recall and rest was $30 \mathrm{~s}$. Because participants were able to finish the BR or FR task within $14 \mathrm{~s}$, there was enough time (more than $16 \mathrm{~s}$ ) for the BOLD signal to recover to the baseline level. Using a video projector, stimuli were presented on a translucent screen placed near the scanner bed, so that the participants could view the stimuli through a mirror placed above their eyes. The fixation-point, the digits, and the cue to instruct participants to recall extended at $2^{\circ} \times 2^{\circ}, 2^{\circ} \times 2^{\circ}$, and $2^{\circ} \times 3^{\circ}$ visual angles on the screen respectively. Each participant completed two scans, one including ten BR trials and the other including ten FR trails. Before each scan, the type of task was told to participants. The order of the scans was counterbalanced among the participants.

\section{MRI data acquisition and processing}

Imaging data were collected on a 1.5 T Philips Infinion MR System (12). A circularly polarized head coil was used, with foam padding to restrict head motion. Functional images were acquired with a $\mathrm{T} 2 *$-weighted

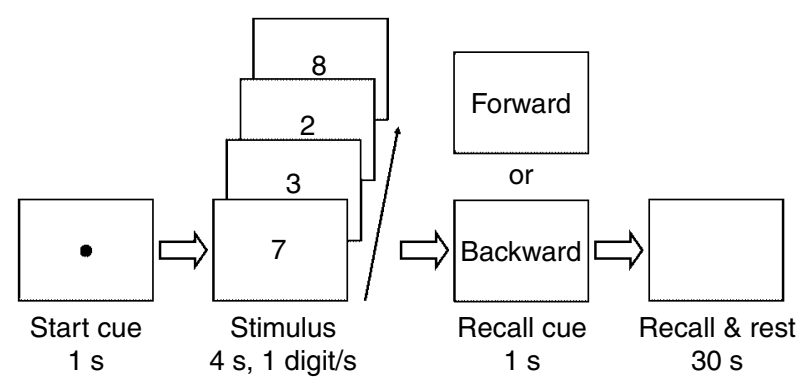

Figure 1 The sample paradigm of the four-digit backward (BR) and forward recall (FR) task. The reaction cues in actual experiments were displayed in Chinese. echo-planar imaging sequence (repetition time $(\mathrm{TR})=$ $2 \mathrm{~s}$, echo time $(\mathrm{TE})=40 \mathrm{~ms}$, field of view $(\mathrm{FOV})=$ $24 \times 24 \mathrm{~cm}^{2}$, matrix $=64 \times 64$, flip angle $=90^{\circ}$ ) with 21 axial slices (slice gap $=1.2 \mathrm{~mm}$, voxel size $=3.75 \times$ $3.75 \times 4 \mathrm{~mm}^{3}$ ) covering the whole cerebrum. Corresponding high-resolution T1-weighted imaging data were also obtained with a spin echo sequence (for anatomical overlay) and a spoiled gradient-recalled echo sequence (for stereotaxic transformation).

MRI data were analyzed using the Analysis of Functional NeuroImages (AFNI) (12, 24-26). For each run, the first three time points were discarded to account for the approach to steady state in the BOLD signal. The raw data were corrected for temporal shifts between slices and head motion, spatially smoothed with a Gaussian kernel (full width at half maximum $=$ $4 \mathrm{~mm}$ ), and temporally normalized (for each voxel, the signal of each image was divided by the temporally averaged signal). Realignment parameters for each participant were carefully examined to ensure that the participant's head movement did not exceed $2 \mathrm{~mm}$.

For each participant, the functional data set of each task (BR or FR) was correlated with a box-car time series (Task vs rest. Twenty different time series were contained. These time series started from the second, third, fourth, and fifth TR of each trial, with widths of one to five TRs, and all convolved with a gamma haemodynamic response function) to generate the individual correlation maps (27). Voxels that were positively/ negatively correlated with the determined box-car time series were defined as activated (TIA)/deactivated (TID) voxels during the task respectively. The fit coefficients, which represented the scaling value required to best fit the reference time series to the observed BOLD signal, were used as a measure of the magnitude of TIA/TID (27). Very large fit coefficients (absolute value $>15$ ) were assumed to correspond to large draining veins and were ignored in the analyses (27).

All individual correlation maps were transformed to the Talairach space (re-sampled voxel size: $3 \times 3 \times$ $3 \mathrm{~mm}^{3}$ ) according to the spatial transformation between the anatomic data and the Talairach space (28). Totally, six maps (controls, pre-treatment patients, and post-treatment patients, during BR or FR respectively) were generated by voxel-wise one-sample $t$-test. The survived clusters were determined by combining individual voxel threshold of $P<0.001$ with a spatial cluster (cluster size from 40 to 49 voxels among the six maps), which yielded a false-positive level of 0.05 over the whole cerebrum according to Monte Carlo simulations conducted with AFNI (26). Clusters that did not reach this significant level were discarded.

The six maps of survived clusters were combined together with a logical 'OR' to locate the regions of interest (ROIs) (24). The ROIs of task-positive network were defined as clusters showing TIA, including the bilateral dorsolateral prefrontal cortices (dIPFC, BA 9), bilateral middle/inferior frontal gyri (M/IFG, BA 
6/9/44), supplementary motor areas (SMAs, BA 6/24, including part of dorsal anterior cingulate cortex), premotor areas (PMA, BA 6), and parietal areas (PA, BA $7 / 39 / 40)$. The ROIs of task-negative network were defined as clusters showing TID, including the bilateral medial prefrontal cortices (mPFC, BA 32/24, including part of rostral anterior cingulate cortex), posterior cingulate cortices (PCC, BA 31/7, including part of precuneus), and inferior parietal lobules (IPL, BA 39). The Talairach's coordinate and voxel size for each ROI were shown in Supplementary Table 1, (see section on supplementary data given at the end of this article). For each task of every participant, fit coefficients of all voxels within each ROI were averaged and dropped into further group level analyses.

\section{Statistical analysis}

Student's $t$-tests for independent or paired samples in this study were used to evaluate the significance of group differences in age, education, serum hormone levels, psychometric evaluation, and fMRI data (described in detail below) with SPSS version 13.0 (SPSS, Chicago, IL, USA). Statistical significance for these analyses was set at $\alpha=0.05$ (all tests were two tailed).

A subtraction method (29) was applied in the group level analysis of fMRI data. In this study, FR was considered as a reference task in which mainly shortterm memory was involved, whereas BR was considered as the true working memory task because it needs more attention and executive processes to generate the reverse sequence $(24,30)$. Because global effects of multiple non-neural factors, such as age-, mood-, disease-, and medication-related changes in neurovascular coupling, may confound the interpretation of differences in the BOLD signal $(31,32)$, systematic differences between conditions in one group versus another are considered to better reflect the specific differences in underlying neural activity (31). Therefore, either TIA or TID during FR was subtracted from that during BR within each ROI to generate the magnitude of BR-FR contrast (brain function contrast during BR and FR), which was used as the index of magnitude of working memory component (the additional attention and executive function involved in BR relative to FR). This index was then compared among the groups (patients (pre-treatment, or post-treatment) versus controls) using independent sample $t$-test or among the patient conditions (pre-treatment versus posttreatment) using paired $t$-test.

\section{Results}

\section{Clinical and psychometric evaluation}

All hypothyroid patients were diagnosed with elevated $\mathrm{TSH}$ and abnormally low $\mathrm{fT}_{4}$ and $\mathrm{fT}_{3}$ levels, while all of which were restored within normal ranges after the ${\mathrm{L}-\mathrm{T}_{4}}_{\text {treatment. The pre-treatment differences }}$ between patients and controls in these serum hormone levels were eliminated after treatment (Table 1). Pretreatment patients showed significantly lower MQ $(P=0.007)$ and marginally significant lower numeric span $(P=0.090)$ than controls, which indicated poor memory states and slight working memory deficit. After treatment, the patients' memory states (including working memory) were significantly ameliorated (pretreatment versus post-treatment, $P<0.016$ ) and reached comparable levels as those of controls (posttreatment patients versus controls, $P>0.483$ ) (Table 1 ).

\section{Neuroimaging}

The recall accuracies for fMRI four-digit BR and FR task were very high in all three groups (controls (BR: $97.50 \% \pm 4.52$; FR: $100 \% \pm 0$ ), pre-treatment (BR: $99.23 \% \pm 2.77$; FR: $100 \% \pm 0$ ), post-treatment (BR: 99.23\% 2.77; FR: 99.23\% \pm 2.77$)$ ), with no significant differences between groups or patient conditions $(P>0.05)$.

\section{Comparisons of working memory component (BR-FR contrast)}

TIA within task-positive network regions TIA was observed during both BR and FR in both patients and controls within the bilateral dIPFC, M/IFG, SMA, PMA and PA (detailed data shown in Supplementary Table 2, see section on supplementary data given at the end of this article).

However, no significant difference in the magnitude of BR-FR contrast of TIA was found within any region between groups (patients versus controls), neither before, nor after treatment. There were no significant differences between the patient conditions (either pretreatment or post-treatment; $P>0.05$ ).

TID within task-negative network regions TID was observed during both BR and FR in both patients and controls within the bilateral mPFC, PCC, and IPL (detailed data shown in Supplementary Table 2, see section on supplementary data given at the end of this article).

Before treatment, significant differences of the magnitude of BR-FR contrast of TID between pretreatment patients and controls were found in the bilateral mPFC (left, $t(1,23)=2.340, P=0.028$; right, $t(1,23)=2.202, P=0.038)$, PCC (left, $t(1,23)=3.038$, $P=0.006$; right, $t(1,23)=2.326, P=0.029)$, and left IPL $(t(1,23)=2.813, P=0.010$; Fig. 2 , Table 2$)$. As a deactivation, the change in TID magnitude is opposite to common activations. Enhanced TID would result in more negative fMRI signals, vice versa. In order to better demonstrate the differences in TID among the groups, 
we intentionally reversed the $y$ axis in Fig. 2. In these regions, an enhanced TID was found during BR relative to FR in controls, whereas a diminished TID was found during $\mathrm{BR}$ relative to $\mathrm{FR}$ in pre-treatment patients (find detailed demonstrations in Supplementary Figure 1, see section on supplementary data given at the end of this article).

After treatment, the pre-treatment differences of the magnitude of BR-FR contrast of TID were no longer detected within the same regions $(P>0.05$; Fig. 2, Table 2). The TID of post-treatment patients had resumed to a similar pattern as that of controls, whilst enhanced TID was found during BR relative to FR (find detailed demonstrations in Supplementary Figure 1, see section on supplementary data given at the end of this article). Furthermore, significant differences between pre-treatment and posttreatment patients were found in the bilateral PCC (left, $t(1,12)=2.484, P=0.029$; right, $t(1,12)=$ $2.219, P=0.047)$, and left IPL $(t(1,12)=2.209$, $P=0.047)$ (Fig. 2, Table 2).

\section{Discussion}

This study intended to explore potential alteration of brain function in hypothyroid patients during working memory processing. Brain function related to working memory was evaluated between groups (patients (pretreatment and post-treatment) versus controls) and patient conditions (pre-treatment versus post-treatment), using BR-FR contrast as an index. Before treatment, the results from WMS-CR revealed significant worse memory states (including slightly working memory deficit) in patients. During the fMRI scanning, the patients (pre-treatment and post-treatment) performed comparably well as controls in the four-digit BR and FR task. No significant difference of BR-FR contrast of TIA between pre-treatment patients and controls was found. However, significant differences of BR-FR contrast of TID between pre-treatment patients and controls were found in the bilateral mPFC, PCC, and left IPL. In these brain areas, the patients showed diminished TID during BR in contrast to FR relative to

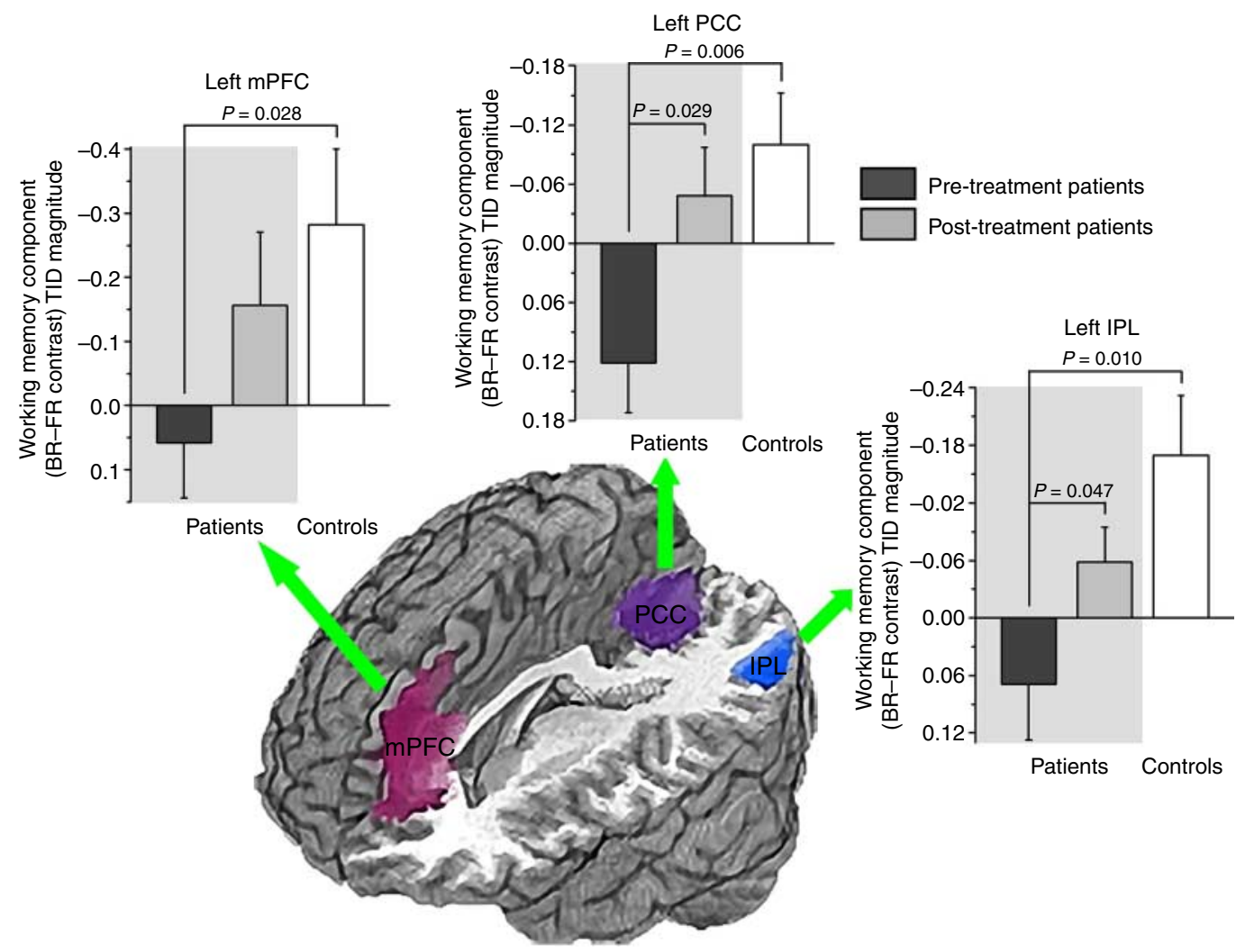

Figure 2 Comparisons of working memory component (BR-FR contrast) related TID within task-negative network between patients with controls. Regions showing TID during the four-digit BR and FR task are demonstrated. mPFC, medial prefrontal cortex; PCC, posterior cingulate cortex; IPL, inferior partial lobule. Only comparisons within the left side of brain regions were shown, whereas those within the right side of brain regions were similar except for the right IPL. Detailed data for both sides could be found in Table 2. In order to better demonstrate the deactivation, $y$ axes were reversed. At diagnosis, significant differences between pre-treatment patients with controls were found in the MPFC, PCC, and IPL. After treatment, no significant difference between post-treatment patients with controls was found in all these brain regions. Significant differences between pre-treatment and post-treatment patients were found in the PCC and IPL. TID magnitude, average fit coefficient. Full colour version of this figure available via http://dx.doi.org/10.1530/EJE-11-0046. 
Table 2 Working memory component (BR-FR) related TID within task-negative network. Significant differences revealed by student's $t$-tests are shown in bold.

\begin{tabular}{|c|c|c|c|c|c|c|}
\hline \multirow[b]{2}{*}{ ROls } & \multicolumn{2}{|c|}{ Patients (mean \pm S.D.) } & \multirow[b]{2}{*}{$\begin{array}{c}\text { Controls } \\
(\text { mean } \pm \text { S.D. })\end{array}$} & \multicolumn{3}{|c|}{ Student's $t$-tests ( $P$ value) } \\
\hline & Pre & Post & & Pre vs Controls & Post vs Controls & Pre vs Post \\
\hline $\begin{array}{l}\text { L-mPFC } \\
\text { R-mPFC } \\
\text { L-PCC } \\
\text { R-PCC } \\
\text { L-IPL }\end{array}$ & $\begin{array}{l}0.06 \pm 0.31 \\
0.08 \pm 0.42 \\
0.12 \pm 0.18 \\
0.14 \pm 0.22 \\
0.07 \pm 0.21\end{array}$ & $\begin{array}{l}-0.16 \pm 0.41 \\
-0.12 \pm 0.34 \\
-0.05 \pm 0.18 \\
-0.02 \pm 0.19 \\
-0.06 \pm 0.13\end{array}$ & $\begin{array}{l}-0.28 \pm 0.41 \\
-0.29 \pm 0.43 \\
-0.10 \pm 0.18 \\
-0.06 \pm 0.21 \\
-0.17 \pm 0.22\end{array}$ & $\begin{array}{l}0.028 \\
0.038 \\
0.006 \\
0.029 \\
0.010\end{array}$ & $\begin{array}{l}0.452 \\
0.280 \\
0.476 \\
0.570 \\
0.129\end{array}$ & $\begin{array}{l}0.154 \\
0.177 \\
\mathbf{0 . 0 2 9} \\
\mathbf{0 . 0 4 7} \\
\mathbf{0 . 0 4 7}\end{array}$ \\
\hline
\end{tabular}

ROI, region of interest; pre, pre-treatment hypothyroid patients; post, post-treatment hypothyroid patients; L, left; R, right; mPFC, medial prefrontal cortex; $\mathrm{PCC}$, posterior cingulate cortex; IPL, inferior partial lobule.

controls. After having been treated with $\mathrm{L}^{-\mathrm{T}_{4}}$ for $\sim 6$ months, the patients showed significantly ameliorated memory states (including working memory), which reached comparable levels as those of controls. In particular, all pre-treatment statistical differences of BR-FR contrast of TID between the two groups were no longer detected, implying a restoring progress of brain functioning within these regions of patients.

Both TIA and TID were observed in both patients and controls performing the four-digit BR and FR task. TIA was found in the task-positive network, including the bilateral dIPFC, M/IFG, SMA, PMA, and PA. These brain areas play a role in the attentional, executive, and mnemonic processes involved in generating and maintaining original/reverse digit sequences (24). Correspondingly, TID was found in the task-negative network, including the bilateral mPFC, PCC, and IPL. These brain areas play a role in the reallocation of mental resources by suppressing less task-relevant cognitive processes (that is how the deactivation comes from) (18-20).

To our knowledge, it is the first time that the alteration of TID has been revealed in patients with thyroid hormone deficiency. Former studies on euthyroid subjects had found that increase of cognitive load was related to enhancement of TID $(19,27,33)$. In particular, TID of bilateral mPFC and left IPL was more sensitive to attention load, whereas the TID of bilateral PCC was sensitive to both attention and mnemonic loads involved in working memory tasks (34). In this study, we found consistent results in controls, although the pre-treatment hypothyroid patients showed diminished TID during BR (high load) contrast to FR (low load) relative to controls. Similar diminishment of TID was also found in several brain diseases and mental disorders, such as age-related cognitive impairment (20), Alzheimer's disease (35), and major depression (36), etc.

The regions showing altered TID in this study, including the bilateral mPFC, PCC, and left IPL, were also considered as parts of the default mode network $(\mathrm{DMN})(18,37)$, which were frequently found activated during rest for their role in 'stimulus-independent thoughts', ranging from internal and external monitoring to mind wandering $(38,39)$. The DMN plays an important role in the pathophysiology of many brain diseases and mental disorders $(39,40)$. However, there is no direct evidence for the mechanism of hypothyroidism-related alteration of TID within DMN regions. Several possibilities should be considered. An interpretation may be that hypothyroidism affects TID through regulating neurotransmitters, such as dopamine. Animal studies showed that hypothyroidism was related to decreased accumulation rate of dopamine (41), and downregulation of dopamine was related to diminished TID (42). Another possibility may be that hypothyroidism alters regional brain perfusion (43) or metabolism (44) within these DMN regions, which results in alteration of TID. Additionally, pathological changes in thyroid autoimmunity $(45,46)$, genetic polymorphisms of deiodinase enzymes and thyroid hormone transporters $(45,47)$, as well as mood disorders associated with hypothyroidism $(7,48-50)$, etc. could also be potential explanations. Further investigations with corresponding approaches are required to test all these possibilities.

In this study, we did not find significant alteration of TIA in pre-treatment patients underlying comparable task performance. However, this result did not suggest that the task-positive network was intact in hypothyroid patients. Altered TIA within task-positive network regions, including the bilateral $\mathrm{dIPFC}, \mathrm{M} / \mathrm{IFG}$, and SMA were found combining with worse task performance in subclinical hypothyroid patients when they were asked to perform a harder working memory task (two-back) during fMRI scanning (12). Therefore, there could have been potential alteration of TIA beyond our present observation, which may be concealed by a potential ceiling effect due to the relatively easy task condition (average accuracies over 95\% were achieved in all groups).

Interestingly, differences in TID were not concealed at the same easy task condition within the same subjects, which may be of potential implications. A necessary precondition for fMRI application is that the participants should perform the task acceptably well during scanning, because they may disengage from the task if it is too hard (51), which would render the corresponding 
fMRI data meaningless. For example, in our previous study, hypothyroid patients were excluded from fMRI investigation because they could not perform the twoback task at an acceptable level (12), although this task was considered to be more sensitive (9). Comparing with hard tasks, easy tasks, i.e. digit span, are more practicable and less restricted by the wide range of cognitive deficit severity in hypothyroid patients $(5,52)$, and could be performed even by patients with relatively severe hypothyroidism. Accordingly, TID during easy task conditions may play a promising role in clinical applications in exploring the pathological cognitive processes in hypothyroidism.

The L- $\mathrm{T}_{4}$ treatment was useful for restoration of both behavior performance and brain function. After the treatment, the memory states (including working memory function) of patients were significantly ameliorated to comparable levels of controls, which were consistent with some previous behavior studies (23, 53). Meanwhile, no significant difference in brain function was detected either in TIA or in TID, which was also in line with former neuroimaging studies (44). Significant differences of TID between pre- and posttreatment patients were found in the bilateral PCC and left IPL, suggesting an overt treatment-related restoration of brain function within these regions.

Hypothyroidism is also frequently associated with mood disorders, mostly depression and anxiety (48-50). Cognitive deficits in hypothyroidism were found in both studies in which mood disturbances were controlled (9, $54)$ or not $(1,2)$. Meanwhile, during working memory processing, both similarity and differences between current findings with those from mood disorder studies were revealed. Depression would result in diminished TID within the mPFC, which is similar to our results, but not within the PCC or IPL (36). In the contrary, anxiety would result in elevated TID within the DMN regions (55). Nevertheless, as the neuropsychological symptoms were not specifically evaluated in this study, the potential role of hypothyroidism-related mood disorders in altered brain function remains to be explored.

Further limitations of the study design should be considered, such as training effect. Previous evidence $(56,57)$ showed that the training-related cerebral activation was found after about 2 weeks of daily working memory practices (over ten times of practices in total) and changed following an inverse U-shaped quadratic function within 4 weeks (56). Accordingly, our findings may not be mainly accounted for the training effects due to both the lack of practices (no specific training between the two experimental sessions) and the relatively long time interval (over 6 months). However, the sample size of this study is relatively limited. Therefore, our results should be viewed as exploratory and the association of thyroid hormone or thyroid autoimmunity with brain function remains to be explored in the future.
In summary, this study provides novel evidence for alteration of brain function in hypothyroid patients during working memory processing. Altered TID within DMN regions was found before treatment but no longer detectable after the $\mathrm{L}_{-} \mathrm{T}_{4}$ treatment. These findings implied potential clinical applications of TID in investigating the pathological cognitive processes in hypothyroidism.

\section{Supplementary data}

This is linked to the online version of the paper at http://dx.doi.org/10. 1530/EJE-11-0046.

\section{Declaration of interest}

There is no conflict of interest that could be perceived as prejudicing the impartiality of the research reported.

\section{Funding}

This work was supported by the National Natural Science Foundation of China (30772301, 30770713, and 30870764), and Ministry of Science and Technology of China (No. 2006CB500705).

\section{Acknowledgements}

We thank Rong Liu, Chun-Lei Liu and Hu-Shen Xu for help with data collection; we also thank Dr De-Lin Sun and Fen Wang for their advice.

\section{References}

1 Bauer M, Goetz T, Glenn T \& Whybrow PC. The thyroid-brain interaction in thyroid disorders and mood disorders. Journal of Neuroendocrinology 200820 1101-1114. (doi:10.1111/j.13652826.2008.01774.x)

2 Davis JD \& Tremont G. Neuropsychiatric aspects of hypothyroidism and treatment reversibility. Minerva Endocrinologica 200732 49-65.

3 Constantinou C, Margarity M \& Valcana T. Region-specific effects of hypothyroidism on the relative expression of thyroid hormone receptors in adult rat brain. Molecular and Cellular Biochemistry 2005278 93-100. (doi:10.1007/s11010-005-6934-z)

4 Broedel O, Eravci M, Fuxius S, Smolarz T, Jeitner A, Grau H, Stoltenburg-Didinger G, Plueckhan $H$, Meinhold $H$ \& Baumgartner A. Effects of hyper- and hypothyroidism on thyroid hormone concentrations in regions of the rat brain. American Journal of Physiology, Endocrinology and Metabolism 2003285 E470-E480. (doi:10.1152/ajpendo.00043.2003)

5 Dugbartey AT. Neurocognitive aspects of hypothyroidism. Archives of Internal Medicine $1998 \mathbf{1 5 8}$ 1413-1418. (doi:10.1001/ archinte.158.13.1413)

6 Lass P, Slawek J, Derejko M \& Rubello D. Neurological and psychiatric disorders in thyroid dysfunctions. The role of nuclear medicine: SPECT and PET imaging. Minerva Endocrinologica 2008 33 75-84.

7 Samuels MH. Cognitive function in untreated hypothyroidism and hyperthyroidism. Current Opinion in Endocrinology, Diabetes, and Obesity 200815 429-433. (doi:10.1097/MED.0b013 e32830eb84c)

8 Bunevicius R \& Prange AJ. Mental improvement after replacement therapy with thyroxine plus triiodothyronine: relationship to cause of hypothyroidism. International Journal of Neuropsychopharmacology 20003 167-174. (doi:10.1017/ S1461145700001826) 
9 Samuels MH, Schuff KG, Carlson NE, Carello P \& Janowsky JS. Health status, mood, and cognition in experimentally induced subclinical hypothyroidism. Journal of Clinical Endocrinology and Metabolism 200792 2545-2551. (doi:10.1210/jc.2007-0011)

10 Baddeley A. Working memory. Science 1992255 556-559. (doi:10.1126/science.1736359)

11 Wickelgren I. Getting a grasp on working memory. Science 1997 275 1580-1582. (doi:10.1126/science.275.5306.1580)

12 Zhu DF, Wang ZX, Zhang DR, Pan ZL, He S, Hu XP, Chen XC \& Zhou JN. FMRI revealed neural substrate for reversible working memory dysfunction in subclinical hypothyroidism. Brain 2006 129 2923-2930. (doi:10.1093/brain/awl215)

13 Sorensen AG. Future prospects for fMRI in the clinic. Journal of Magnetic Resonance Imaging 200623 941-944. (doi:10.1002/ jmri.20589)

14 Detre JA \& Floyd TF. Functional MRI and its applications to the clinical neurosciences. Neuroscientist 2001 7 64-79. (doi:10. 1177/107385840100700110)

15 Hennig J, Speck O, Koch MA \& Weiller C. Functional magnetic resonance imaging: a review of methodological aspects and clinical applications. Journal of Magnetic Resonance Imaging 2003 18 1-15. (doi:10.1002/jmri.10330)

16 Smith EE, Jonides J, Marshuetz C \& Koeppe RA. Components of verbal working memory: evidence from neuroimaging. PNAS 199895 876-882. (doi:10.1073/pnas.95.3.876)

17 D'Esposito M, Postle BR \& Rypma B. Prefrontal cortical contributions to working memory: evidence from event-related fMRI studies. Experimental Brain Research 2000133 3-11. (doi:10.1007/s002210000395)

18 Mazoyer B, Zago L, Mellet E, Bricogne S, Etard O, Houde O, Crivello F, Joliot M, Petit L \& Tzourio-Mazoyer N. Cortical networks for working memory and executive functions sustain the conscious resting state in man. Brain Research Bulletin 200154 287-298. (doi:10.1016/S0361-9230(00)00437-8)

19 Tomasi D, Ernst T, Caparelli EC \& Chang L. Common deactivation patterns during working memory and visual attention tasks: an intra-subject fMRI study at 4 Tesla. Human Brain Mapping 2006 27 694-705. (doi:10.1002/hbm.20211)

20 Sambataro F, Murty VP, Callicott JH, Tan HY, Das S, Weinberger DR \& Mattay VS. Age-related alterations in default mode network: impact on working memory performance. Neurobiology of Aging 201031 839-852. (doi:10.1016/j.neurobiolaging.2008.05.022)

21 Gong Y, Wang D \& Deng J. Handbook of Wechsler Memory Scale-Revised. Changsha, China: Bulletin of Human Medical College, 1989.

22 Wechsler D. Wechsler Memory Scale-Revised. New York: Psychological Corporation, 1987.

23 Wekking EM, Appelhof BC, Fliers E, Schene AH, Huyser J, Tijssen JG \& Wiersinga WM. Cognitive functioning and wellbeing in euthyroid patients on thyroxine replacement therapy for primary hypothyroidism. European Journal of Endocrinology 2005 153 747-753. (doi:10.1530/eje.1.02025)

24 Sun X, Zhang X, Chen X, Zhang P, Bao M, Zhang D, Chen J, He S \& $\mathrm{Hu} X$. Age-dependent brain activation during forward and backward digit recall revealed by fMRI. NeuroImage 200526 36-47. (doi:10.1016/j.neuroimage.2005.01.022)

25 Cox RW. AFNI: software for analysis and visualization of functional magnetic resonance neuroimages. Computers and Biomedical Research 199629 162-173. (doi:10.1006/cbmr.1996.0014)

26 Ma N, Liu Y, Li N, Wang CX, Zhang H, Jiang XF, Xu HS, Fu XM, $\mathrm{Hu} \mathrm{X} \&$ Z Zhang DR. Addiction related alteration in resting-state brain connectivity. NeuroImage 200949 738-744. (doi:10.1016/ j.neuroimage.2009.08.037)

27 McKiernan KA, Kaufman JN, Kucera-Thompson J \& Binder JR. A parametric manipulation of factors affecting task-induced deactivation in functional neuroimaging. Journal of Cognitive Neuroscience $2003 \quad 15 \quad 394-408 . \quad$ (doi:10.1162/089892903 321593117)

28 Talairach J, Tournoux P. Co-Planar Stereotaxic Atlas of the Human Brain. New York: Thieme Medical Publishers, 1988.
29 Smith YR, Love T, Persad CC, Tkaczyk A, Nichols TE \& Zubieta JK. Impact of combined estradiol and norethindrone therapy on visuospatial working memory assessed by functional magnetic resonance imaging. Journal of Clinical Endocrinology and Metabolism 200691 4476-4481. (doi:10.1210/jc.2006-0907)

30 Hedden $\mathrm{T} \&$ Gabrieli JD. Insights into the ageing mind: a view from cognitive neuroscience. Nature Reviews. Neuroscience 20045 87-96. (doi:10.1038/nrn1323)

31 Buckner RL, Snyder AZ, Sanders AL, Raichle ME \& Morris JC. Functional brain imaging of young, nondemented, and demented older adults. Journal of Cognitive Neuroscience 200012 (Supplement 2) 24-34. (doi:10.1162/089892900564046)

32 D'Esposito M, Deouell LY \& Gazzaley A. Alterations in the BOLD fMRI signal with ageing and disease: a challenge for neuroimaging. Nature Reviews. Neuroscience 2003 4 863-872. (doi:10. 1038/nrn1246)

33 Chee MW \& Choo WC. Functional imaging of working memory after $24 \mathrm{hr}$ of total sleep deprivation. Journal of Neuroscience 2004 24 4560-4567. (doi:10.1523/JNEUROSCI.0007-04.2004)

34 Mayer JS, Roebroeck A, Maurer K \& Linden DE. Specialization in the default mode: task-induced brain deactivations dissociate between visual working memory and attention. Human Brain Mapping 201031 126-139. (doi:10.1002/hbm.20850)

35 Rombouts SA, Barkhof F, Goekoop R, Stam CJ \& Scheltens P. Altered resting state networks in mild cognitive impairment and mild Alzheimer's disease: an fMRI study. Human Brain Mapping 200526 231-239. (doi:10.1002/hbm.20160)

36 Rose EJ, Simonotto E \& Ebmeier KP. Limbic over-activity in depression during preserved performance on the n-back task. NeuroImage 200629 203-215. (doi:10.1016/j.neuroimage. 2005.07.002)

37 Raichle ME, MacLeod AM, Snyder AZ, Powers WJ, Gusnard DA \& Shulman GL. A default mode of brain function. PNAS 200198 676-682. (doi:10.1073/pnas.98.2.676)

38 Gusnard DA \& Raichle ME. Searching for a baseline: functional imaging and the resting human brain. Nature Reviews. Neuroscience 20012 685-694. (doi:10.1038/35094500)

39 Buckner RL, Andrews-Hanna JR \& Schacter DL. The brain's default network: anatomy, function, and relevance to disease. Annals of the New York Academy of Sciences 20081124 1-38. (doi:10.1196/annals.1440.011)

40 Broyd SJ, Demanuele C, Debener S, Helps SK, James CJ \& SonugaBarke EJ. Default-mode brain dysfunction in mental disorders: a systematic review. Neuroscience and Biobehavioral Reviews 200933 279-296. (doi:10.1016/j.neubiorev.2008.09.002)

41 Ito JM, Valcana T \& Timiras PS. Effect of hypo- and hyperthyroidism on regional monoamine metabolism in the adult rat brain. Neuroendocrinology 197724 55-64. (doi:10.1159/000122696)

42 Tomasi D, Volkow ND, Wang R, Telang F, Wang GJ, Chang L, Ernst T \& Fowler JS. Dopamine transporters in striatum correlate with deactivation in the default mode network during visuospatial attention. PLoS ONE 20094 e6102. (doi:10.1371/journal.pone. 0006102 )

43 Krausz Y, Freedman N, Lester H, Newman JP, Barkai G, Bocher M, Chisin R \& Bonne O. Regional cerebral blood flow in patients with mild hypothyroidism. Journal of Nuclear Medicine $2004 \mathbf{4 5}$ 1712-1715.

44 Bauer M, Silverman DH, Schlagenhauf F, London ED, Geist CL, van Herle K, Rasgon N, Martinez D, Miller K, van Herle A, Berman SM, Phelps ME \& Whybrow PC. Brain glucose metabolism in hypothyroidism: a positron emission tomography study before and after thyroid hormone replacement therapy. Journal of Clinical Endocrinology and Metabolism 200994 2922-2929. (doi:10. 1210/jc.2008-2235)

45 Bunevicius $\mathrm{R}$ \& Prange AJ Jr. Thyroid disease and mental disorders: cause and effect or only comorbidity? Current Opinion in Psychiatry 201023 363-368. (doi:10.1097/YCO. Ob013e3283387b50)

46 Ferracci F \& Carnevale A. The neurological disorder associated with thyroid autoimmunity. Journal of Neurology 2006253 975-984. (doi:10.1007/s00415-006-0170-7) 
47 Wiersinga WM. Do we need still more trials on $\mathrm{T}_{4}$ and $\mathrm{T}_{3}$ combination therapy in hypothyroidism? European Journal of Endocrinology 2009161 955-959. (doi:10.1530/EJE-09-0879)

48 Constant EL, de Volder AG, Ivanoiu A, Bol A, Labar D, Seghers A, Cosnard G, Melin J \& Daumerie C. Cerebral blood flow and glucose metabolism in hypothyroidism: a positron emission tomography study. Journal of Clinical Endocrinology and Metabolism $2001 \mathbf{8 6}$ 3864-3870. (doi:10.1210/jc.86.8.3864)

49 Schraml FV, Beason-Held LL, Fletcher DW \& Brown BP. Cerebral accumulation of Tc-99m ethyl cysteinate dimer (ECD) in severe, transient hypothyroidism. Journal of Cerebral Blood Flow and Metabolism 200626 321-329. (doi:10.1038/sj.jcbfm.9600191)

50 Constant EL, Adam S, Seron X, Bruyer R, Seghers A \& Daumerie C. Anxiety and depression, attention, and executive functions in hypothyroidism. Journal of the International Neuropsychological Society 2005 11 535-544. (doi:10.1017/S1355617705050642)

51 Callicott JH, Mattay VS, Bertolino A, Finn K, Coppola R, Frank JA, Goldberg TE \& Weinberger DR. Physiological characteristics of capacity constraints in working memory as revealed by functional MRI. Cerebral Cortex 19999 20-26. (doi:10.1093/cercor/9.1.20)

52 Roberts CG \& Ladenson PW. Hypothyroidism. Lancet 2004363 793-803. (doi:10.1016/S0140-6736(04)15696-1)

53 Miller KJ, Parsons TD, Whybrow PC, van Herle K, Rasgon N, van Herle A, Martinez D, Silverman DH \& Bauer M. Memory improvement with treatment of hypothyroidism. International Journal of Neuroscience $2006 \mathbf{1 1 6} 895-906$. (doi:10.1080/ 00207450600550154 )

54 Burmeister LA, Ganguli M, Dodge HH, Toczek T, DeKosky ST \& Nebes RD. Hypothyroidism and cognition: preliminary evidence for a specific defect in memory. Thyroid $2001111177-1185$. (doi:10.1089/10507250152741037)

55 Fales CL, Barch DM, Burgess GC, Schaefer A, Mennin DS, Gray JR \& Braver TS. Anxiety and cognitive efficiency: differential modulation of transient and sustained neural activity during a working memory task. Cognitive, Affective and Behavioral Neuroscience 2008 8 239-253. (doi:10.3758/CABN.8.3.239)

56 Hempel A, Giesel FL, Garcia Caraballo NM, Amann M, Meyer H, Wustenberg T, Essig M \& Schroder J. Plasticity of cortical activation related to working memory during training. American Journal of Psychiatry 2004161 745-747. (doi:10.1176/appi.ajp. 161.4.745)

57 Olesen PJ, Westerberg H \& Klingberg T. Increased prefrontal and parietal activity after training of working memory. Nature Neuroscience 20047 75-79. (doi:10.1038/nn1165)

Received 19 March 2011

Accepted 5 April 2011 